\title{
A Survey on Consumers' Perception of Illegally Reused Standing Wreaths and the Introduction of New Standing Wreaths
}

\author{
Song Kwon' ${ }^{1}$ Ju Hyun Ryu ${ }^{2}$ and Hye Jin Kwon ${ }^{3 *}$ \\ ${ }^{1}$ Research Institute of Agriculture and Life Science, Seoul National University, Seoul 08826, Korea \\ ${ }^{2}$ Siheung City Agriculture Technology Center, Siheung City Hall, Siheung 14998, Korea \\ ${ }^{3}$ Division of Smart Horticulture, Yonam College, Cheonan 31005, Korea
}

\begin{abstract}
The distribution of standing wreaths has reached about 7.1 million per year and the size of the standing wreath market is estimated to be at least 700 billion won. Among them, 20 to 30 percent are estimated to be reused, resulting in damage to flowerfarms' sales of 110 to 160 billion won per year. Due to the unique characteristics of conventional 3-tier standing wreaths in terms of transportation and installation, companies that lack design expertise have monopolized local distribution markets, and the issue of illegally using standing wreaths has been raised. These issues have highlighted the need for developing new designs and establishing a distribution system to expand the consumption of flowers. This study was carried out to identify consumers' perception of reused standing wreaths and the current systems introduced to prevent the distribution of reused standing wreaths such as the real-name standing wreath production system, the authenticity certificate system, and new standing wreaths. The results of a consumer survey showed that consumers' perception of reused standing wreaths has been high and that reused standing wreaths need to be actively regulated to establish fair trade order and protect consumers' rights. Although the effectiveness of the real-name system, the authenticity certificate system, and new standing wreaths was found to be insufficient, the introduction of these systems was essential and necessary for continuous promotion through broadcasting media.
\end{abstract}

Keywords: certification system, distribution system, floricultural industry development, flower consumption, labelling

\section{Introduction}

The number of standing wreaths distributed annually is about 7,100,000, and the size of the standing wreath market is estimated to be over 700 billion won (Han et al., 2016). Among them, 20 to 30 percent are estimated to be reused. Reused standing wreaths are produced by collecting wreaths used once, removing withered or unmerchantable flowers and adding some fresh flowers for resale. The resale of reused standing wreaths has resulted in damage to flower farms' sales of 110 160 billion won annually (Park et al., 2013). Due to the unique characteristics of conventional 3-tier standing wreaths

\footnotetext{
This study was supported by the Agricultural Biotechnology Development Program of the Korea Institute of Planning and Evaluation for Technology in food, Agriculture and Forestry under the Ministry for Food, Agriculture, Forestry and Fisheries (317069-2).

Received: November 12, 2018, Revised: November 20, 2018, Accepted: December 4, 2018

First author: Song Kwon, E-mail: freesia77@empal.com, ORCID: 0000-0002-3190-7217

*Corresponding author: Hye Jin Kwon, E-mail: hjkwon@yonam.ac.kr, ORCID: 0000-0002-3540-7850
} 
in terms of transportation and installation, companies that lack design expertise have monopolized local distribution markets, and issues of illegally using standing wreaths and consumers' negative perception of reused standing wreaths have been raised (Lim et al., 2013; Yang and Park, 2015). These issues have highlighted the necessity of analyzing problems in the distribution market of standing wreaths that account for a large portion of domestic cut flower consumption, developing new standing wreath designs and establishing a distribution system that can expand the consumption of flowers. Several systems have been implemented to prevent the distribution of reused standing wreaths, and, among them, the real-name standing wreath production system is a system in which information on the production of standing wreaths is marked on the label of standing wreaths to strengthen the responsibilities and guarantee of producers for the quality of standing wreaths. Under the authenticity certificate system, a certificate sticker for certifying that new flowers are used only without reusing flowers is produced and attached to the lower part of a standing wreath to ensure consumers can identify the authenticity of the standing wreath they bought. The system was introduced to prevent the reuse of flowers, but it has been difficult to implement the system (Park et al., 2015). New standing wreaths, unlike conventional standing wreaths, are a new concept of standing wreaths made of $100 \%$ fresh flowers, and the government has actively promoted new standing wreaths in order to expand the consumption of flowers, but their base has yet to be expanded. Against this backdrop, this study aimed to identify prerequisites for preventing producers from distributing reused standing flowers and for introducing new standing wreaths through a survey on consumers' perception of reused standing wreaths and new standing wreaths, and to suggest measures to improve the distribution of standing flowers in order to expand flower consumption and establish healthy flower culture.

\section{Methods}

\section{Subjects}

A questionnaire survey was performed among 113 people in order to examine consumers' perception of reused standing wreaths and systems that have been implemented to prevent the distribution of reused standing wreaths.

\section{Survey Methods}

A questionnaire survey was carried out between August and November, 2018, and the questionnaire was composed of questions about the consumption of standing wreaths, understanding of reused standing wreaths, understanding of the real-name standing wreath production system and the authenticity certificate system, and new standing wreaths. Each item's percentage was expressed as the ratio of the total number of surveyed subjects to the number of those who answered each question. The characteristics of respondents were surveyed by gender and age group. The frequency of the collected data were analyzed using SPSS.

\section{Results and Discussion}

\section{General Characteristics of Subjects}

Frequency analysis was performed to examine the general characteristics of subjects, and the results showed that the share of females (54.9\%) was higher than that of males (45.1\%). The share of those in their 40s (25.7\%) and 60s or older (20.3\%) was relatively higher than that of other age groups. To the question about experience of purchasing standing wreaths, $44.2 \%$ of the subjects answered "yes" and, to the question about the purchasing price of a standing wreath, $46.0 \%$ answered that it was lower than 100,000 won. The majority of the subjects surveyed in this study were found to prefer 
purchasing standing wreaths on the Internet (52.0\%). The general characteristics of the surveyed subjects were as shown in Table 1.

\section{Understanding of Reused Standing Wreaths}

The results of the questionnaire survey on consumers' perception showed that $65.5 \%$ of the total respondents were aware of the practice of reusing standing wreaths for wedding or funeral (Figure 1A), and that, to the question about the route to obtain information on reused standing wreaths, $43.2 \%$ answered "press reports including TV," followed by "Internet (21.6\%)" (Figure 1B). The share of those who agreed with the labelling of reused standing wreaths was the highest (60.2\%), and 18.6\% of the total respondents disagreed with it (Figure 1C). Those who agreed with the labelling of reused standing wreaths were asked about their reason, and the share of those who answered "to establish fair trade order (39.7\%)" and "to meet consumers' right to know (30.9\%)" was relatively higher than other reasons (Table 2). Those who disagreed with the labelling of reused standing wreaths were asked about their reason, and the share of those who answered "the price of standing wreaths can be increased due to the cost of labelling passed on to consumers" was the highest (47.6\%), followed by "there is no system that can root out the practice of trading standing wreaths that violate the labelling system (28.6\%)" (Table 3). Park et al. (2015) also showed similar results that many consumers obtained

Table 1. Experimental design

\begin{tabular}{|c|c|c|c|}
\hline & Variable & Frequency (n) & Percentage $(\%)$ \\
\hline \multirow[t]{3}{*}{ Gender } & Male & 51 & 45.1 \\
\hline & Female & 62 & 54.9 \\
\hline & Total & 113 & 100.0 \\
\hline \multirow[t]{7}{*}{ Age (years) } & $<20$ & 2 & 1.8 \\
\hline & $20-29$ & 17 & 15.0 \\
\hline & $30-39$ & 21 & 18.6 \\
\hline & $40-49$ & 29 & 25.7 \\
\hline & $50-59$ & 21 & 18.6 \\
\hline & $\geq 60$ & 23 & 20.3 \\
\hline & Total & 113 & 100.0 \\
\hline \multirow[t]{3}{*}{ Experience of purchasing standing wreaths } & Yes & 50 & 44.2 \\
\hline & No & 63 & 55.8 \\
\hline & Total & 113 & 100.0 \\
\hline \multirow[t]{6}{*}{ Purchasing price of standing wreaths (thousand) } & $<50$ & 9 & 18.0 \\
\hline & $50-99$ & 23 & 46.0 \\
\hline & $100-149$ & 13 & 26.0 \\
\hline & $150-199$ & 3 & 6.0 \\
\hline & $\geq 200$ & 2 & 4.0 \\
\hline & Total & 50 & 100.0 \\
\hline \multirow[t]{6}{*}{ Route to purchase standing wreaths } & Flower shop & 11 & 22.0 \\
\hline & Telephone & 7 & 14.0 \\
\hline & Chain & 6 & 12.0 \\
\hline & Internet & 26 & 52.0 \\
\hline & Others & 0 & 0 \\
\hline & Total & 50 & 100.0 \\
\hline
\end{tabular}



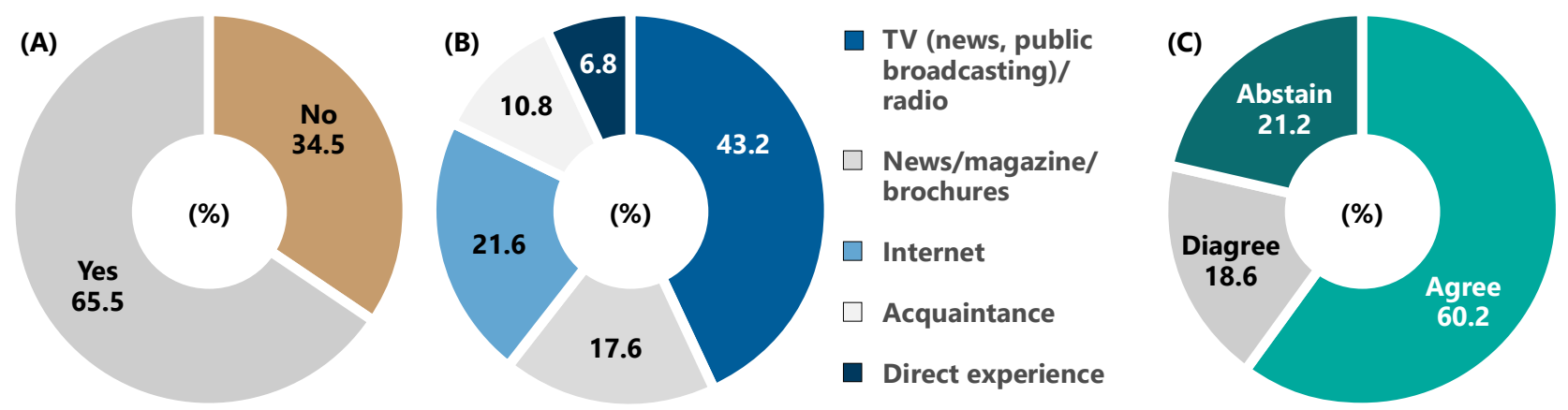

Figure 1. Consumers' perception (A), route to obtain information (B), and views on the labelling system (C) of reused standing wreaths.

Table 2. Reasons to agree with the labelling of reused standing wreaths

\begin{tabular}{lc}
\hline \multicolumn{1}{c}{ Variable } & Percentage (\%) \\
\hline To establish fair trade order & 39.7 \\
To purchase reusable standing wreaths at a low cost & 16.2 \\
To contribute to an increase in the income of flower farms & 13.2 \\
To meet consumers' right to know & 30.9 \\
Total & 100.0 \\
\hline
\end{tabular}

Table 3. Reasons to disagree with the labelling of reused standing wreaths

\begin{tabular}{lr}
\hline \multicolumn{1}{c}{ Variable } & Percentage (\%) \\
\hline The negative images of the system could reduce the value of the entire market of standing wreaths. & 14.3 \\
The price of standing wreaths can be increased due to the cost of labelling passed on to consumers. & 47.6 \\
The current certification system is not reliable. & 9.5 \\
There is no system that can root out the practice of trading standing wreaths that violate the labelling system & 28.6 \\
Total & 100.0 \\
\hline
\end{tabular}

information on reused standing wreaths through broadcasting media including TV. Many consumers answered "highly agree" to the question about whether they thought selling reused standing wreaths is an act of disrupting fair trade order, and, considering that, it seems to be necessary to continuously raise the issue of reusing standing wreaths.

\section{Understanding of the Real-name Standing Wreath Production System}

Those who were aware of the real-name standing wreath production system introduced to prevent the reuse of standing wreaths accounted for $11.5 \%$ (Figure 2A), and most of them obtained the information through "press reports (53.8\%)" (Figure $2 \mathrm{~B}$ ). The majority of the respondents agreed with the introduction of the real-name standing wreath production system (61.1\%), and those who disagreed with the system accounted for 15.9\% (Figure 2C). Those who agreed with the introduction of the real-name standing wreath production system were asked about their reason, and the share of those who answered "to restore the trust relationship with consumers (42.0\%)" and "to meet consumers' right to know (33.3\%)" was high (Table 4). Those who disagreed with the introduction of the real-name standing wreath production system were asked about their reason, and the share of those who answered "the price of standing wreaths can be increased due to the cost of labelling passed on to consumers" was the highest (50.0\%) (Table 5). 

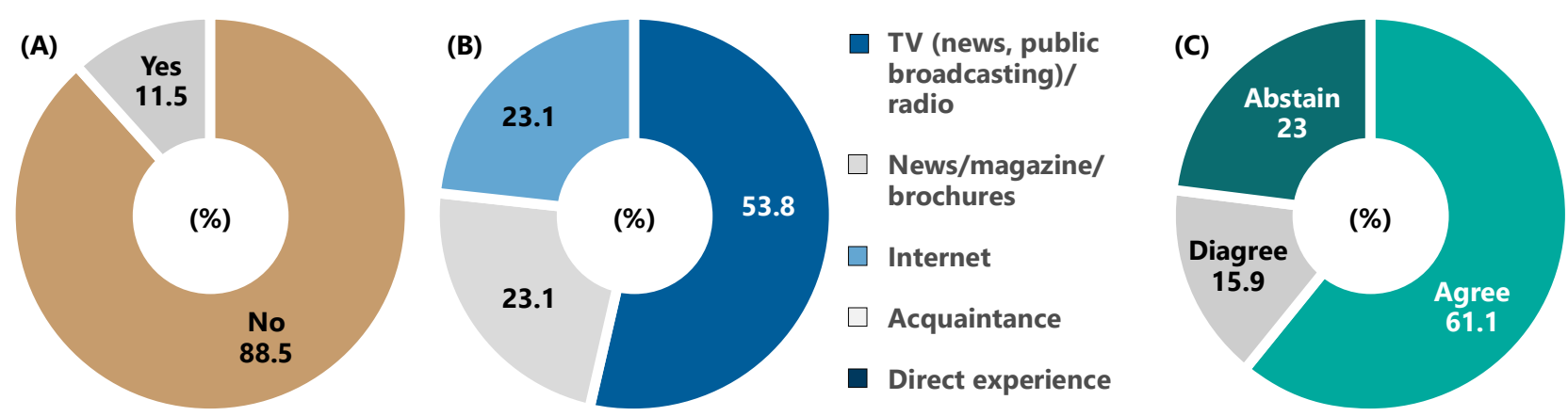

Figure 2. Consumers' perception (A), route to obtain information (B), and views on the introduction (C) of the real-name standing wreath production system.

Table 4. Reasons to agree with the real-name standing wreath production system

\begin{tabular}{lr}
\hline \multicolumn{1}{c}{ Variable } & Percentage $(\%)$ \\
\hline To prevent the reuse of standing wreaths & 10.2 \\
To purchase trustworthy standing wreaths that use fresh flowers & 14.5 \\
To restore the trust relationship with consumers & 42.0 \\
To meet consumers' right to know & 33.3 \\
\hline Total & 100.0 \\
\hline
\end{tabular}

Table 5. Reasons to disagree with the real-name standing wreath production system

\begin{tabular}{lr}
\hline \multicolumn{1}{c}{ Variable } & Percentage (\%) \\
\hline Companies that produce standing wreaths can be directly damaged when issues arise. & 11.1 \\
The price of standing wreaths can be increased due to the cost of labelling passed on to consumers. & 50.0 \\
There is no specific standard to regulated reused standing wreaths. & 16.7 \\
There is no system that can root out the practice of trading wreaths that violate the labelling system. & 22.2 \\
Total & 100.0 \\
\hline
\end{tabular}

\section{Understanding of the Authenticity Certificate System}

Those who were aware of the authenticity certificate system introduced to root out the practice of reusing standing wreaths accounted for $13.3 \%$ (Figure 3A), and most of them obtained the information through "press reports 40.0\%" (Figure 3B). The majority of the respondents agreed with the introduction of the authenticity certificate system (63.7\%), and those who disagreed with the system accounted for $17.7 \%$ (Figure 3C). Those who agreed with the authenticity certificate system were asked about their reason, and the share of those who answered "to purchase trustworthy standing wreaths that use fresh flowers only (38.9\%)" and "to sell products that consumers can trust (25.0\%)" was high (Table 6). Those who disagreed with the authenticity certificate system were asked about their reason, and the share of those who answered "the price of standing wreaths can be increased due to the cost of labelling passed on to consumers (45.0\%)" was the highest (Table 7). Lim et al. (2013) showed that many people answered that "fresh flowers should be used instead of artificial flowers without vitality (24.7\%)" for standing flowers in weddings, funerals and events," and, considering that, it seems to be necessary to make efforts to distribute quality standing wreaths in order to restore consumers' trust. 

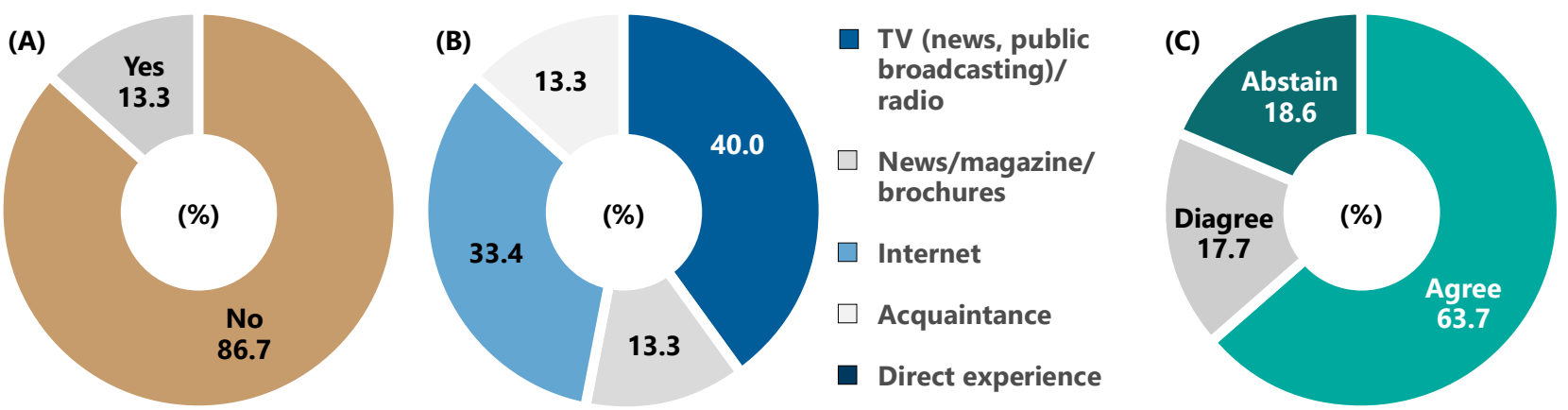

Figure 3. Consumers' perception (A), route to obtain information (B), and views on the introduction (C) of the authenticity certification system.

Table 6. Reasons to agree with the authenticity certification system

\begin{tabular}{lc}
\hline \multicolumn{1}{c}{ Variable } & Percentage $(\%)$ \\
\hline To prevent the reuse of standing wreaths & 15.3 \\
To purchase trustworthy standing wreaths that use fresh flowers & 38.9 \\
To sell products that consumers can trust & 25.0 \\
To meet consumers' right to know & 20.8 \\
Total & 100.0 \\
\hline
\end{tabular}

Table 7. Reasons to disagree with the authenticity certification system

\begin{tabular}{lr}
\hline \multicolumn{1}{c}{ Variable } & Percentage (\%) \\
\hline Companies that produce standing wreaths can be directly damaged when issues arise. & 10.0 \\
The price of standing wreaths can be increased due to the cost of labelling passed on to consumers. & 45.0 \\
There is no specific standard to regulated reused standing wreaths. & 15.0 \\
There is no system that can root out the practice of trading wreaths that violate the labelling system. & 30.0 \\
Total & 100.0 \\
\hline
\end{tabular}

\section{Understanding of New Standing Wreaths}

Those who were aware of new standing wreaths made of $100 \%$ fresh flowers that can prevent the reuse of flowers unlike conventional standing wreaths accounted for $10.6 \%$ (Figure 4A), and most of them obtained the information through "press reports (41.7\%)" (Figure 4B). The majority of the respondents answered that they were willing to purchase new standing wreaths (53.1\%), while those who disagreed accounted for 46.9\% (Figure 4C). Those who answered that they were willing to purchase new standing wreaths were asked about their reason, and the share of those who answered "to select designs that are not uniform but give esthetic beauty (36.7\%)" and "to purchase trustworthy standing wreaths that use fresh flowers only (28.3\%)" was high (Table 8). Those who answered that they were not willing to purchase new standing wreaths were asked about their reason, and the share of those who answered "the price of standing wreaths will be higher than that of conventional 3-tier standing wreaths (37.7\%)" and "they feel uncomfortable about new models due to familiarity with conventional 3-tier standing wreaths (32.1\%)" was high (Table 9). Han et al. (2016) also showed that very few respondents were aware of new standing wreaths (6\%). However, when their willing to pay (WTP) that included the emotional and cultural values of new standing wreaths was surveyed using the contingent valuation method (CVM), they were found to be willing to additionally pay for new standing wreaths. Considering this, continuously promoting new 

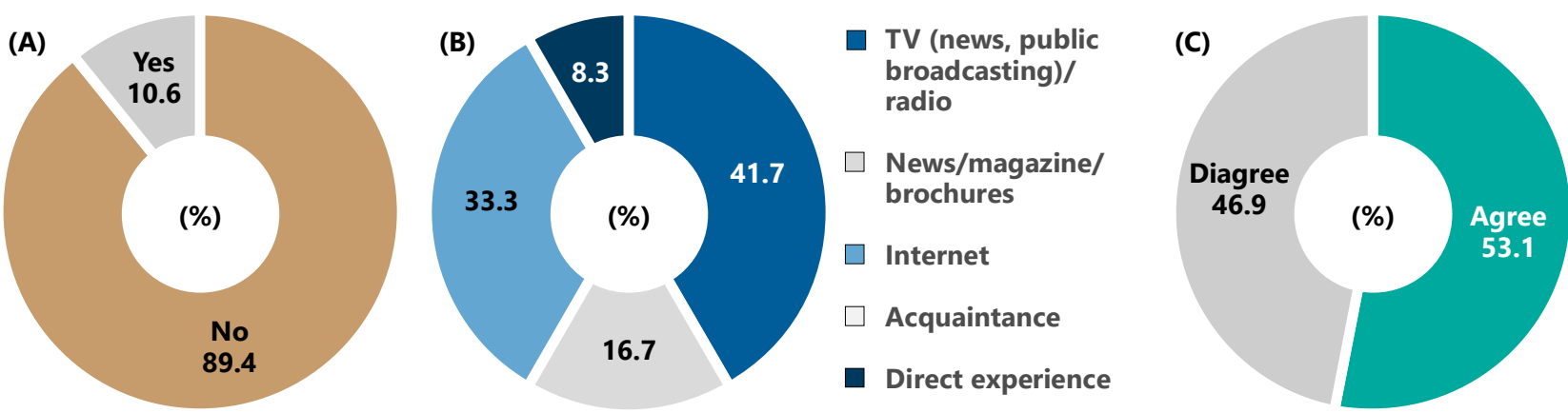

Figure 4. Consumers' perception (A), route to obtain information (B), and views on the introduction (C) of new standing wreaths.

Table 8. Reasons to agree with purchasing new standing wreaths

\begin{tabular}{lc}
\hline \multicolumn{1}{c}{ Variable } & Percentage (\%) \\
\hline To establish a sound distribution system that can prevent the reuse of standing wreaths & 18.3 \\
To purchase trustworthy standing wreaths that use fresh flowers only & 28.3 \\
To select designs that are not uniform but give esthetic beauty & 36.7 \\
To improve the overall culture of standing wreaths & 16.7 \\
Total & 100.0 \\
\hline
\end{tabular}

Table 9. Reasons to disagree with purchasing new standing wreaths

\begin{tabular}{lc}
\hline \multicolumn{1}{c}{ Variable } & Percentage (\%) \\
\hline The price of standing wreaths will be higher than that of conventional standing wreaths. & 37.7 \\
They feel uncomfortable about new models due to familiarity with conventional standing wreaths. & 32.1 \\
The conventional standing wreath distribution system is convenient. & 13.2 \\
They are not suitable for the current culture of celebration and consolation. & 17.0 \\
Total & 100.0 \\
\hline
\end{tabular}

standing wreaths through broadcasting media is expected to be a key factor for the popularization of new standing wreaths.

\section{Conclusion}

The number of standing wreaths distributed annually is about 7,100,000, and the size of the standing wreath market is estimated to be over 700 billion won. Among them, 20 to 30 percent are estimated to be reused, and the estimated amount of damage to flower farms sales of 110 160 billion won annually. Due to the unique characteristics of conventional 3-tier standing wreaths in terms of transportation and installation, companies that lack design expertise have monopolized local distribution markets, and issues of illegally using standing wreaths and consumers' negative perception of reused standing wreaths have been raised. These issues have highlighted the necessity of analyzing problems in the distribution market of standing wreaths that account for a large portion of domestic cut flower consumption, developing new standing wreath designs and establishing a distribution system that can expand the consumption of flowers. With the aim of examining consumers' perception of reused standing wreaths, and systems that had been implemented to prevent the distribution of reused standing wreaths, 113 people were surveyed through a questionnaire composed of questions about the labelling of 
reused standing wreaths, the real-name standing wreath production system, the authenticity certificate system, and the introduction of new standing wreaths. The results of the survey showed that consumers' perception of reused standing wreaths was high, and that reused standing wreaths need to be actively regulated in order to establish fair trade order and protect consumers' rights. The effectiveness of the real-name standing wreath production system, the authenticity certificate system and new standing wreaths was also examined. The results showed that consumers' perception of them was very low, and it seems that the introduction of these systems is essential, and that they need to be continuously promoted through broadcasting media in order to prevent reusing standing wreaths.

\section{References}

Han, K.W., E.J. Kim, and C.H. Pak. 2016. Current status of use standing wreath (event flower) and improvement plan for floricultural industry development. Korean J. Hortic. Sci. Technol. 34(Suppl I):52-53.

Lim, J.H., I. Baek, M. Sim, and J. Suh. 2013. A Study for current status of cut flower distribution and for a way of promoting flower consumption (Report 11-1543000-000312-01). Sejong, Korea: Ministry of Agriculture, Food and Rural Affairs.

Park, C.H., K. Han, J. Hong, and E. Kim. 2015. Current status of use standing wreath and artificial flowers, problem analysis, and improvement plan for floricultural industry development. Seoul, Korea: Korean Floricultural Producers Council.

Park, K.H., S. Huh, and D. Lee. 2013. Strategic development of floricultural industry for stable consumption and export industrialization (Research reports R 704-1). p. 141. Naju, Korea: Korea Rural Economic Institute.

Yang, J.H. and N.B. Park. 2015. Vitalization study on floricultural industry by analyzing domestic flower consumption realities. Pract. Agric. Fish. Res., KNCAF. 17(1):21-43. 